\title{
Operative Treatment with a Laparotomy for Anorectal Problems Arising from a Self-Inserted Foreign Body
}

\author{
Seung-Bum Ryoo ${ }^{1}$, Heung-Kwon $\mathrm{Oh}^{1}$, Heon-Kyun $\mathrm{Ha}^{1}$, Eun Kyung Choe ${ }^{1,2}$, Sang Hui Moon ${ }^{1}$, Kyu Joo Park ${ }^{1}$ \\ ${ }^{1}$ Division of Colorectal Surgery, Department of Surgery, Seoul National University College of Medicine, Seoul; ${ }^{2}$ Healthcare Research Institute, \\ Seoul National University Hospital Healthcare System Gangnam Center, Seoul, Korea
}

An anorectal foreign body can cause serious complications such as incontinence, rectal perforation, peritonitis, or pelvic abscess, so it should be managed immediately. We experienced two cases of operative treatment for a self-inserted anorectal foreign body. In one, the foreign body could not be removed as it was completely impacted in the anal canal. We failed to remove it through the anus. A laparotomy and removal of the foreign body was performed by using an incision on the rectum. Primary colsure and a sigmoid loop colostomy were done. A colostomy take-down was done after three months. The other was a rectal perforation from anal masturbation with a plastic device. We performed primary repair of the perforated rectosigmoid colon, and we didea sigmoid loop colostom. A colostomy take-down was done three months later. Immediate and proper treatment for a self-inserted anorectal foreign body is important to prevent severe complications, and we report successful surgical treatments for problems caused by anorectal foreign bodies.

Keywords: Anal canal; Foreign bodies; Laparotomy

\section{INTRODUCTION}

Although cases of and anally-inserted foreign object causing problems are not common, if proper treatment is not administered in emergency situations, complications of anorectal dysfunction, fecal incontinence, perforation, peritonitis, pelvic abscess, etc. may occur. In addition, due to the fact that most patients conceal their conditions or do not seek medical help initially, diagnosis and treatment are delayed, and serious damage to the anorectum may result. With these patients, first, through anorectum secretion and anoscope exams, the existence and the location of the foreign object are ascertained; then, removal of the foreign object is attempted through the anus, for which local anesthesia or general anesthesia may sometimes be administered. However, in cases where the re-

Received: July 7, 2011 • Revised: August 12, 2011

Accepted: August 12, 2011

Correspondence to: Kyu Joo Park, M.D.

Department of Surgery, Seoul National University Hospital, Seoul National University College of Medicine, 101 Daehak-ro, Jongno-gu, Seoul 110-744, Korea

Tel: +82-2-2072-2901, Fax: +82-2-766-3975

E-mail:kjparkmd@plaza.snu.ac.kr

(c) 2012 The Korean Society of Coloproctology

This is an open-access article distributed under the terms of the Creative Commons Attribution NonCommercial License (http://creativecommons.org/licenses/by-nc/3.0) which permits unrestricted noncommercial use, distribution, and reproduction in any medium, provided the original work is properly cited. moval attempt is not successful or in cases with serious damages, such as perforations or complications like pelvic abscesses, a laparotomy may be necessary. Although many cases of damages from anal insertion of vibrators during sexual intercourse or from the mentally insane or prison inmates anally inserting foreign objects are being reported in the West, these kinds of reports are rare in Korea. However, as the lifestyle is becoming more westernized, these types of patients are expected to increase in the future [1-4]. As such, the authors of this study wanted to report two cases of patients undergoing laparotomies at the Seoul National University Hospital due to self-inserted foreign objects in the anorectum.

\section{CASE REPORT}

\section{Case 1}

A 35-year-old male patient visited the hospital because he was unable to remove an anally-inserted foreign object. According to the patient, one day before visiting the hospital, he had been "drinking and playing with friends when he anally inserted a glass ball," and because he couldn't remove the glass ball, he visited the hospital. The first hospital he visited attempted to remove the object, but failed; therefore, he underwent a first laparotomy, but the finding was that because the object was completely stuck in his anorectum, it could not be removed. Thus, the patient was moved to our hospital for a second surgery. 
Volume 28, Number 1, 2012

The findings during the exam at our hospital showed that the object was completely stuck in the lower rectum and anus, and the patient complained of lower abdominal pain and abdominal rebound tenderness. There were no hemodynamic findings or any blood abnormalities. In a simple abdominal shooting, there was a finding of a ball-shaped foreign object impacted in the pelvic cavity (Fig. 1); through the anus, the removal of this foreign object was attempted. Visibility in the anal canal was attained through the use of an anal examination retractor, and the object was located, after which a removal attempt was made by hand. However, it was not easy to remove the object, which was completely stuck in the anus and the lower rectum. Because the patient complained of excruciating pain, the attempt to remove the object through the anus

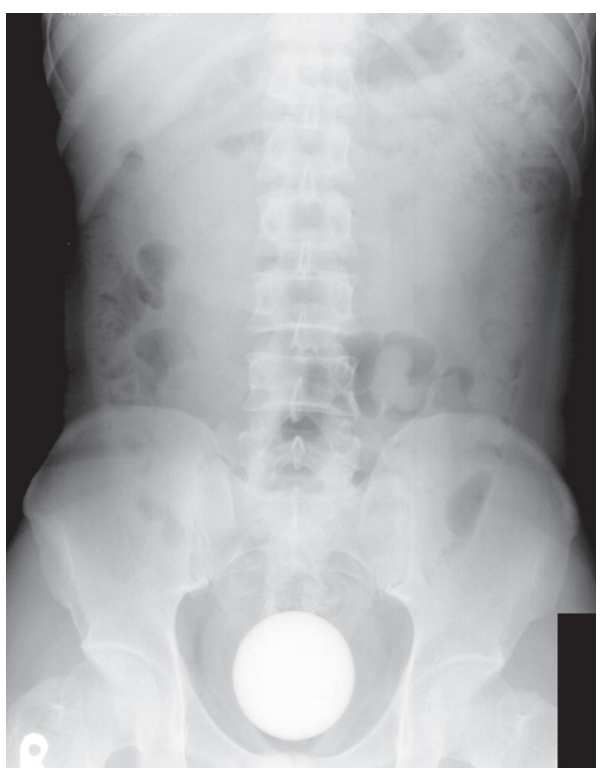

Fig. 1. Simple $\mathrm{X}$-ray image of an impacted foreign body at the pelvis. was stopped; instead, surgical treatment was attempted.

Under general anesthesia, an attempt to remove through the anus was made again, but it failed, so a laparotomy was commenced. During the laparotomy, the foreign object was found to be completely stuck in the anus and lower rectum, and the proximal colon was occluded. From within the peritoneal cavity, an attempt was made to carefully push the object in the direction of the rectal canal, but it was again not possible to remove it through the anus. To avoid any additional damage to the rectum and anus, we detached and mobilized the rectum and gently pushed the object into the peritoneal cavity, and after which we made a linear incision in the upper rectum and removed the object. A simple suture was made (Fig. 2), but the incision was too large. Due to the fact that the object had not been removed immediately and a considerable amount of time had elapsed since the object's insertion, much fecal matter was found to have accumulated in the rectum and the proximal colon, and an edema was found in the rectum. Therefore, additionally, a sigmoid colostomy was performed to prevent any complications that might occur in the peritoneal and the pelvic cavities from future leakage.

After the surgery, the patient recovered without complications, and although a mild ulceration in the rectal mucosa was found during the outpatient follow-up observation period, the condition improved without any special treatment, and there was no damage to the anal sphincter. Hence, after verifying about 3 months later, through radiation imaging test, that there was no leakage in the incision or rectal damage, we performed a recovery procedure for the sigmoid colon.

\section{Case 2}

A 37-year-old male patient was admitted to the hospital with anal bleeding. The symptoms had started after he had masturbated using an apparatus about $20-\mathrm{cm}$ long in his anus on the early morn-
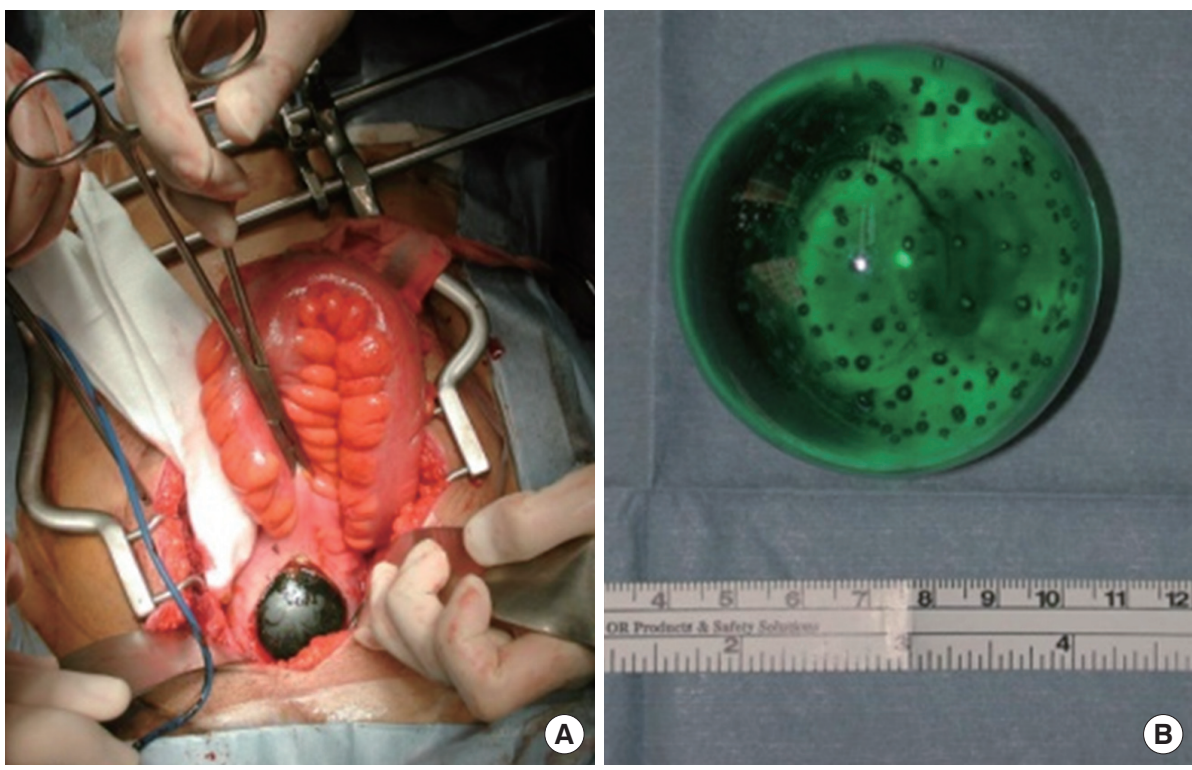

Fig. 2. (A) The upper rectum was incised for removal of a foreign body. (B) Self-inserted glass ball with a round shape and a diameter of about $7 \mathrm{~cm}$. 


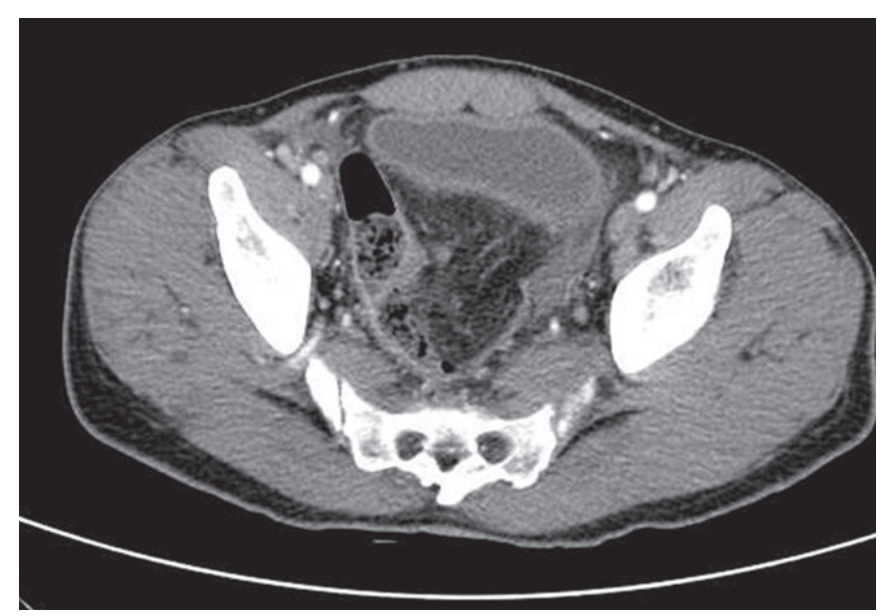

Fig. 3. Computed tomographic image of the rectal perforation and the omental herniation.

ing of the day he was admitted; afterwards, because there were symptoms as if a part of his colon had sprung out through his anus, he visited the emergency room. The patient was bisexual and, according to his medical history, he had contracted human immunodeficiency virus and had been receiving drug treatment through an internal medicine department for infection for 6 years. He had also undergone chemotherapy for Burkitt's lymphoma.

During an anorectal exam conducted at our hospital, a tender fat-like tissue was felt, there was abdominal pain and abdominal rebound tenderness in the entire abdomen, and the patient had a mild fever. There were no abnormalities in the blood test findings. Although no abnormalities were found in the simple abdominal shooting, findings of perforations in the sigmoid colon and of a herniated omentum were found (Fig. 3).

Thus, the patient underwent an emergency laparotomy, the herniated omentum was repaired back into the abdominal cavity via manual reduction and removed, and the perforated area of the sigmoid colon was initially sutured, after which a sigmoid colostomy was performed (Fig. 4). The patient recovered without any problems and was discharged. Three months later, a recovery procedure was performed for the sigmoid colon.

\section{DISCUSSION}

Cases where foreign objects inserted through the anus cause problems in the anorectum are reported to be caused by sexual acts, sexual abuse, self-harming behavior, various accidents, such as traffic accidents, firearm incidents, etc., and the insertion of medical equipment, such as thermometers or enema apparatus. In the West, particularly, foreign objects used in sexual acts by homosexuals and bisexuals or in masturbation causing damage to the anorectum are reported as the most common cases. In addition, children, as victims of sexual abuse, are admitted to hospital emergency rooms for damage sustained in the anorectum, and cases of

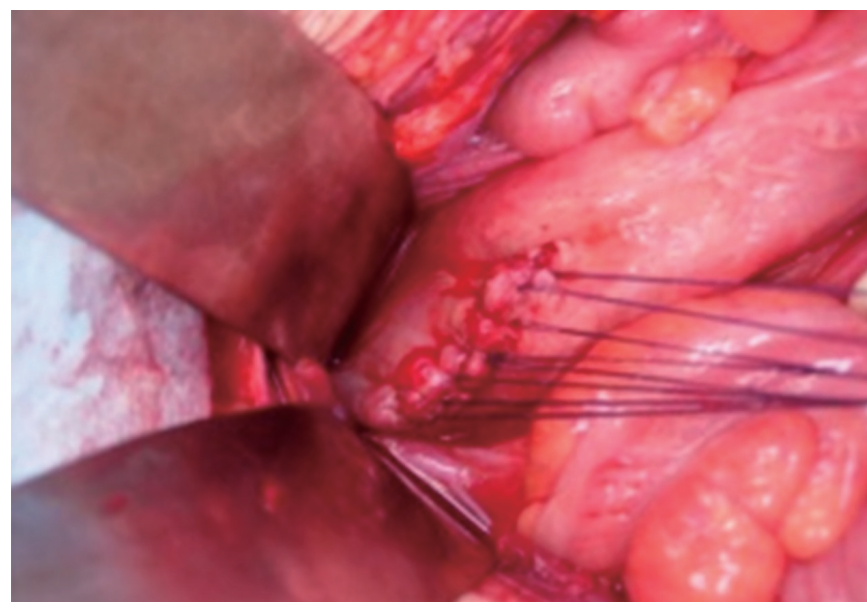

Fig. 4. A primary closure was performed on the rectosigmoid junction.

prison inmates, mentally insane persons, or alcoholics sustaining damages to the anorectum from self-harming acts or accidents are being reported $[1,2]$. In Korea, up until now, reports of these types of cases have been rare [3, 4]; however, due to the westernization of life styles and diverse sexual behaviors, these types of patients are expected to steadily increase. In our case 1, the patient had inserted a foreign object through the anus for entertainment purposes, and in our case 2 , a bisexual patient had sustained a perforated rectum resulting from using a vibrator in a sexual act; thus, patients sustaining injuries to the anorectum caused by these kinds of acts are expected to increase in the future.

Diseases can occur due to foreign objects being inserted into the anorectum as well as foreign objects being orally ingested, passing through the proximal gastrointestinal tract, and getting stuck in the anorectum. Cases of orally-ingested fish bones, toothpicks, etc. getting stuck in the rectal canal and causing acute anal fissures, fistulas, abscesses, etc. are numerous. In Korea, rather than cases of anally-inserted foreign objects, a larger number of incidents where orally-ingested objects cause anal diseases have been reported [5]. The authors of this study reported, in their study of various anal diseases due to orally-ingested objects, that the objects in the anorectum not only cause acute anal pain and anal fissure that is accompanied by abscess and pain in the proximal areas around the anus but also can become aggravating factors of a chronic fistula if not appropriately treated during the acute stages [6].

Many symptoms can be seen in patients who are admitted to the hospital due to problems with foreign objects inserted through the anus. Symptoms may not show initially, and other symptoms such as gas problems, constipation, mucous stools, anal pain, and lower abdominal pain can occur. However, patients tend to resolve the situation by themselves and not to seek medical help; even when they do seek help at a hospital, due to their shame and guilt, they often deny or do not give accurate information about acts of anally inserting foreign objects, thereby causing further delays in receiv- 
ing proper treatment. In cases like these, serious complications in the anorectum from foreign objects may result, and anal sphincter damage or rectal perforations may cause anal bleeding and peritonitis, thereby requiring admission to hospital. In fact, in some cases, patients develop pelvic abscess or sepsis, requiring admission to hospital. When a patient is admitted to the hospital due to atypical symptoms in the anorectum and when symptoms from foreign objects are suspected based on listening to medical history or a secretion exam, then by first conducting a simple abdomen shooting, the presence and location of a foreign object can be ascertained. However, when the objects are not transparent to radiation, they may not be ascertainable through a simple abdominal shooting. Thus, so careful attention must be given to the medical history, and accurate anorectum secretion and anoscope examinations would be important. When peritonitis or pelvic abscess is suspected due to severe abdominal pain, abdominal computed tomography can be performed to ascertain the location of the abscess and the progression of perforations [1-4].

When a foreign object inside the anorectum is verified, an attempt can be made first to remove the object through the anus. However, removal can be difficult due to the structures of the anatomical anorectal angle and the sacral curvature and due to spasms in the anal sphincter caused by pain. An attempt can be made by relaxing the anal sphincter as much as possible with local anesthesia and by carefully applying pressure to the abdomen and removing the object through the anus. In addition, in the operating room under general anesthesia, an attempt can be made to remove the object through the anus by relaxing the anal sphincter as much as possible. It wouldn't hurt to try removing it by using an endoscope. In cases of small foreign objects partially blocking the anorectum, attempts to remove the object can be carefully made by using an endoscope deployed carefully, by passing a catheter, and by inflating a balloon $[7,8]$. Additionally, there have been reports of attempts using forceps or cutters, which are used in obstetrics and plastic surgery, to remove foreign objects $[9,10]$.

In cases where object removal attempts through the anus fail or complications cause anorectal perforations or pelvic abscesses, a laparotomy may be necessary. When a laparotomy is used to remove a foreign object, first, squeezing or milking the anorectum from the abdominal side and pushing the object through the anus can be tried. However, serious injuries to the rectum and the anal canal can occur if excessive force is applied, so such attempts must be made carefully. If such attempts fail, then an incision may need to be made to the rectum or the colon to remove the object. During the surgery, a sigmoid colostomy may be performed to prevent future complications, which is determined by considering the location and the condition of the damaged colon, the degree of proximal invasion, the elapsed time after the damage, the degree of contamination in the abdominal cavity, etc. When there are complications such as rectal perforations or pelvic abscess, via laparotomy, initial suture or removal of damaged colon, a sigmoid colostomy or a Hartmann surgical procedure would need to be performed [1-4]. In our case 1, although several attempts had been made to remove the object through the anus both in the emergency room and the operating room, eventually a laparotomy was necessary. Even with a laparotomy, the object could not be pushed out through the anus, so the object was pushed into the upper rectum, and incisions were made to remove the object, after which a sigmoid colostomy was performed. In our case 2 , because infection in the areas proximal to the rectal perforations was not severe, an initial suture was made; then, a sigmoid colostomy was performed.

After the anally-inserted foreign object has been removed, for confirming the presence and the extent of damage to the colon mucosa, sigmoid colonoscopy can be helpful. When damage to the anal sphincter I suspected, it would need to be verified through anal functionality tests and anal ultrasound. Even after the removal of foreign objects through a laparotomy, continual follow-up is necessary, and anal functions, as well as the presence and the progression of complications, should be monitored. Also, for highrisk patients with the risk or recurrence, psychiatric counseling and treatment can also be important [1-4].

In cases of admission to the hospital with problems caused by self-inserted foreign objects through the anus, initially, knowledge of the medical history in detail and anorectal secretion and anoscope examinations are important; through simple X-ray tests, the location and the type of a foreign object would need to be ascertained, and removal attempts through the anus would quickly need to be made. If these attempts fail or accompanying complications such as rectal perforations and pelvic abscess are present, then a laparotomy would need to be conducted to prevent the progression to serious complications, including sepsis.

In Korea, cases of patients undergoing surgery for complications caused by anally-inserted foreign objects are rather rare. Thus, the two successful cases of treatment through a laparotomy, as performed by the authors of this study, are reported.

\section{CONFLICT OF INTEREST}

No potential conflict of interest relevant to this article was reported.

\section{REFERENCES}

1. Rodríguez-Hermosa JI, Codina-Cazador A, Ruiz B, Sirvent JM, Roig J, Farrés R. Management of foreign bodies in the rectum. Colorectal Dis 2007;9:543-8.

2. Yacobi Y, Tsivian A, Sidi AA. Emergent and surgical interventions for injuries associated with eroticism: a review. J Trauma 2007;62: 1522-30.

3. Choi KD. Foreign bodies in the anorectum: Report of five cases and a review of literature. J Korean Soc Coloproctol 1990;6:55-62.

4. Choi PW, Lee JM, Heo TG, Park JH, Lee MS, Kim CN, et al. Rectal foreign body (glass cup) extracted by laparotomy. J Korean Surg Soc 2008; 74:448-51.

5. Choi DH. Acute anal pain due to ingested bone fragments. J Korean 
Soc Coloproctol 2008;24:51-7.

6. Kim H, Ryoo S, Choe EK, Kim D, Lee D, Park KJ. Anal diseases from ingested foreign bodies. J Korean Soc Coloproctol 2009;25: 387-92.

7. Singaporewalla RM, Tan DE, Tan TK. Use of endoscopic snare to extract a large rectosigmoid foreign body with review of literature. Surg Laparosc Endosc Percutan Tech 2007;17:145-8.

8. Humes D, Lobo DN. Removal of a rectal foreign body by using a
Foley catheter passed through a rigid sigmoidoscope. Gastrointest Endosc 2005;62:610.

9. Golden T, Wangensteen SL. Letter: Rectal foreign-body forceps. JAMA 1973;226:1358.

10. Levin SE, Cooperman H, Freilich M, Lomas M. The use of a curved uterine vulsellum for removal of rectal foreign bodies: report of a case. Dis Colon Rectum 1977;20:532-3. 